\title{
Effects of Balneotherapy and Physical Therapy on Sleep Quality in Patients With Osteoarthritis Aged 50 to 85 Years
}

\author{
Erkan KAYA, ${ }^{1}$ Cengiz KAPLAN, ${ }^{2}$ Alparslan Bayram ÇARLI, ${ }^{1}$ Ümit GÜZELKÜÇÜK ${ }^{3}$ \\ ${ }^{1}$ Department of Physical Medicine and Rehabilitation, Bursa Military Hospital, Bursa, Turkey \\ ${ }^{2}$ Department of Neurology, Bursa Military Hospital, Bursa, Turkey \\ ${ }^{3}$ Department of Physical Medicine and Rehabilitation, GATA TSK Physical Therapy and Rehabilitation Center, Ankara, Turkey
}

\begin{abstract}
Objectives: This study aims to investigate the effect of balneotherapy (BT) and physical therapy (PT) on sleep quality in patients with knee osteoarthritis (OA) aged 50 to 85 years.

Patients and methods: A total of 199 patients (76 males, 123 females; mean age $67.8 \pm 7.3$ years; range 50 to 85 years) suffering from knee OA (Kellgren-Lawrence grade 2-3) for more than six months were enrolled. Sleep and functional status were assessed at baseline and after 19 sessions of BT and 15 sessions of PT by using Pittsburgh Sleep Quality Index and Western Ontario and McMaster Universities Osteoarthritis Index, respectively. Results: A high prevalence of abnormal sleep quality in patients with knee OA was observed. The most common abnormality was sleep fragmentation (71\%), with an increased sleep disturbance score. Patients reported significantly improved sleep, pain, stiffness, and functional status after BT and PT.

Conclusion: Balneotherapy and PT improved self-reported sleep and functional status in patients with OA aged 50 to 85 years. We may conclude that BT and PT, which are used in the treatment of OA, not only reduce nocturnal pain, but also improve sleep quality.

Keywords: Balneotherapy; knee osteoarthritis; sleep disturbance; sleep quality.
\end{abstract}

Osteoarthritis (OA), the most common chronic condition and leading cause of disability worldwide, is a degenerative joint disease characterized by pain and dysfunction due to progressive and irreversible loss of joint cartilage. ${ }^{1}$ It can be defined by radiological abnormalities with or without clinical findings. Osteophytes and joint space narrowing usually affect interphalangeal joints of the hands, knees, and hips. ${ }^{2}$

Pain, depression, and sleep disturbance (SLD) are common complaints associated with OA. Patients with OA report problems with sleep onset (31\%), sleep maintenance (81\%), and early morning awakenings (51\%). ${ }^{3}$ The prevalence of nocturnal knee pain and sleep problems increase with the severity of OA. ${ }^{4}$
There is no specific treatment to repair damaged cartilage in OA. The goal of treatment in $\mathrm{OA}$ is to reduce joint pain and inflammation while improving and maintaining joint function. Some patients may benefit from conservative treatments such as rest, exercise, physical therapy (PT), and balneotherapy (BT). BT involves treating health problems by bathing, usually in hot springs. It can help treating a number of health conditions including arthritis, respiratory disorders, and high blood pressure. ${ }^{5}$ But the literature is poor regarding the effects of $\mathrm{BT}$ and $\mathrm{PT}$ on the sleep quality. Thus, in this study, we aimed to investigate the effects of BT and PT on sleep quality in patients with OA aged 50 to 85 years. It is hypothesized that BT and PT result in improved sleep quality by means of relieving nocturnal knee pain. 


\section{PATIENTS AND METHODS}

This study included 199 patients (76 males, 123 females; mean age $67.8 \pm 7.3$ years; range 50 to 85 years) suffering from knee OA for more than six months who attended to the Physical Medicine and Rehabilitation Department of Bursa Military Hospital between December 2011 and June 2012. All patients' informed consents were obtained. The study was approved by our local research ethics board. Patients were evaluated according to the results of clinical examination, plain X-ray findings, and routine laboratory parameters. OA of the knee was defined as the presence of radiographic changes (joint space narrowing with osteophyte, with or without cyst formation, sclerosis) together with pain. KellgrenLawrence grading system was used to assess radiographic severity of knee OA. ${ }^{6}$ Knee OA was recorded as present if Kellgren-Lawrence grade was 2 or higher. Pain was evaluated on a $100 \mathrm{~mm}$ visual analog scale. All patients had pain during most painful knee movement between 50-70 $\mathrm{mm}$ on visual analog scale. Histories of underlying inflammatory arthropathy, psychiatric/hematological/neurological disorder, hyperuricemia and/or intra-articular corticosteroid injection within the last three months were the exclusion criteria. Each patient completed a questionnaire, providing details regarding demographics, medical history, and sleep characteristics. All patients were treated with one cure (19 days) of BT and 15 sessions PT (ultrasound, hot pack, transcutaneous electrical nerve stimulation, and mud pack). BT was applied in thermo-mineral water pools for 20 minutes with a temperature of $39{ }^{\circ} \mathrm{C}$. The water had a low mineral density with a concentration of $623 \mathrm{mg} / \mathrm{L}$, and none of the minerals were above the accepted threshold levels. Sleep and functional status were assessed at baseline and after treatments of BT and PT by using Pittsburgh Sleep Quality Index (PSQI) and Western Ontario and McMaster Universities Osteoarthritis Index (WOMAC). Functional disability was assessed using WOMAC which produces scores for three subscales: pain, stiffness, and physical function. ${ }^{7}$ Sleep quality and patterns were assessed by using PSQI. PSQI is an effective instrument used to measure the quality and patterns of sleep in older patients. It differentiates "poor" from "good" sleep by measuring seven domains: subjective sleep quality, sleep latency, sleep duration, habitual sleep efficiency, sleep disturbances, use of sleep medication, and daytime dysfunction over the last month. Scoring of the answers is based on a 0 to 3 scale. A global sum of $\geq 5$ indicates poor sleeper. $^{8}$

\section{Statistical analysis}

Statistical analysis was conducted using SPSS for Windows, version 15.0 software program (SPSS Inc., Chicago, IL, USA). The baseline characteristics were shown as the mean \pm standard deviation for continuous data (e.g., age and duration of disease) and $\mathrm{n}(\%)$ for categorical data (e.g. sex). The mean differences from baseline values to the end of treatment were examined using a paired $\mathrm{t}$ test. The level of significance was $\mathrm{p}<0.05$.

\section{RESULTS}

There was no significant difference between the patients who continued and those who dropped out regarding any of the demographic variables (all $\mathrm{p}>0.05$ ), or in sleep, pain stiffness and physical functioning (all $\mathrm{p}>0.05$ ).

Western Ontario and McMaster Universities Osteoarthritis Index showed significant reduction in pain at one-month compared with baseline $(p<0.05)$. Patients scored higher on the PSQI at baseline than at one-month. Table 1 shows WOMAC and PSQI scores of the participants before and after BT and PT. In general, participants reported improvement for each of the component scores; i.e., scored lower at onemonth than baseline, with significant less severe sleep problems at one-month on all domains (all $\mathrm{p}<0.05$ ), except for daytime dysfunction and use of sleep medication.

\section{DISCUSSION}

This study indicates that BT and PT are effective on knee $\mathrm{OA}$ in terms of pain alleviation and improved sleep quality. To our knowledge, this study is one of the largest and most rigorous trials of the efficacy of BT and PT on sleep.

With its various kinds of interventions, balneology or medical hydrology has been an important modality in the field of medicine, which 


\begin{tabular}{|c|c|c|c|}
\hline & Before therapy $(n=199)$ & After therapy $(n=199)$ & \\
\hline & Mean \pm SD & Mean \pm SD & $p$ \\
\hline WOMAC (Pain) & $13.3 \pm 2.3$ & $10.0 \pm 3.1$ & $<0.001$ \\
\hline WOMAC (Stiffness) & $3.9 \pm 1.7$ & $3.3 \pm 2.0$ & $<0.001$ \\
\hline WOMAC (Function) & $35.5 \pm 13.3$ & $28.9 \pm 14.1$ & $<0.001$ \\
\hline PSQI & $7.5 \pm 3.8$ & $6.4 \pm 3.2$ & $<0.001$ \\
\hline Sleep duration & $0.7 \pm 0.9$ & $0.6 \pm 0.7$ & 0.055 \\
\hline Sleep disturbance & $1.7 \pm 0.7$ & $1.6 \pm 0.7$ & 0.666 \\
\hline Sleep latency & $1.4 \pm 0.8$ & $1.4 \pm 0.8$ & 0.359 \\
\hline Daytime dysfunction & $0.9 \pm 0.8$ & $0.7 \pm 0.8$ & 0.016 \\
\hline Habitual sleep efficiency & $0.9 \pm 1.0$ & $0.6 \pm 0.8$ & 0.002 \\
\hline Subjective sleep quality & $1.2 \pm 0.7$ & $1.0 \pm 0.6$ & $<0.001$ \\
\hline Use of sleep medication & $0.5 \pm 0.9$ & $0.4 \pm 0.8$ & 0.082 \\
\hline
\end{tabular}

includes prevention, treatment, and rehabilitation of a large number of health conditions. The main indication for BT in Turkey is rheumatic disorders (degenerative joint diseases, soft tissue disorders, and rarely inflammatory rheumatic diseases).

Pain is the most important feature of OA. However, the mechanisms causing nocturnal knee pain remain unclear, even though many people with knee OA experience it. It is documented that knee OA patients have synovitis even in the early stages of the disease and its prevalence and number of regions increase with the severity of OA. ${ }^{9}$ More regions with synovitis may be related to an increasing prevalence of nocturnal pain. ${ }^{4}$ Previous studies have shown that BT is effective on pain in OA. ${ }^{10-12}$ Wigler et al. ${ }^{10}$ reported that the index of disease severity score was better in patients with OA having daily thermal mineral water baths. Moreover, the well-being status remained for 16 weeks. The beneficial effects or mechanism of action of BT has not been fully understood yet. It can be claimed that these benefits result from the combination of chemical and thermal effects. ${ }^{11,12}$ Although there is little evidence, considering the chemical effect, one might expect that organic substances or minerals are absorbed through the skin during BT. ${ }^{13}$ Extensibility of collagen-rich tissues may increase with thermal stimulation. Because of the increased extensibility of collagenrich tissues, the range of motion of joints improves, pain diminishes, and muscle spasm relieves. The analgesic effect of heat may be due to increased beta-endorphin concentration. ${ }^{14}$
It has been postulated that human skin can release significant amounts of opioid peptides, modifying the threshold of pain under different stimuli, such as heat or ultraviolet radiation. Furthermore, heat may increase secretion of cortisol and catecholamines by thermal stress, thus having an anti-inflammatory effect. ${ }^{15}$ To sum, without ignoring the possible contribution of the chemical substances, we suggest that the observed improvements in the clinical variables in this study were mainly due to the thermal effect.

Sleep quality is a major concern regarding patients with $\mathrm{OA}$; as a matter of fact, $60 \%$ of people with OA reports pain during the night. ${ }^{16}$ In fact, pain secondary to $\mathrm{OA}$ is the most common factor predicting SLD in patients. It is well established that pain hinders sleep and disturbed sleep lowers pain threshold. ${ }^{17-19}$ Whether SLD precedes or follows pain onset is unclear, but reciprocal effects are likely. ${ }^{17}$ Even after treatment with anti-inflammatory medications, patients with OA show significantly greater objective SLD, as compared with age-matched control subjects. Vitiello et al. ${ }^{20}$ reported that BT improved both immediate and long-term self-reported sleep quality in a sample of older patients with $\mathrm{OA}$ and comorbid insomnia. The other major finding of their study is that BT without specifically addressing pain management appeared to reduce both immediate and long-term reported pain. ${ }^{21-23}$ Nevertheless, given the likely reciprocal effects between pain and SLD, distinguishing unique causal pathways is difficult. Chronic pain initiates 
and exacerbates SLD; disturbed sleep in turn maintains and exacerbates chronic pain and related dysfunction. ${ }^{18,19,24}$ The question is whether an intervention that improves sleep in individuals with disturbed sleep and comorbid pain state, such as OA, might reduce pain as well.

This study has some potential limitations. Firstly, participants were recruited primarily through government hospital health reports for BT from all around the country and they might not be representative of all patients with OA of the knee. The other criticism is the impossibility of exclusion of the effects of the BT environment from the study setting. It is often stated that staying at a resort hotel provides a positive placebo effect. And maybe the most important limitation of the study is the lack of a control group.

Balneotherapy and PT were not tested before in a well-controlled study of individuals with sleep disorder and comorbid chronic illnesses such as OA. BT and PT improved immediate self-reported sleep quality in this sample of older patients with $\mathrm{OA}$. These results are unique in demonstrating the effects of BT and PT on sleep disorders. The current study suggests that, in addition to decreasing pain in older patients with OA, and even without directly addressing sleep disorder management, BT and PT appear to improve sleep quality too.

\section{Declaration of conflicting interests}

The authors declared no conflicts of interest with respect to the authorship and/or publication of this article.

\section{Funding}

The authors received no financial support for the research and/or authorship of this article.

\section{REFERENCES}

1. Bodur H. Current review on osteoarthritis in Turkey and the world; epidemiology and socioeconomic aspect. Turk J Geriatr 2011;14:7-14.

2. Abad VC, Sarinas PS, Guilleminault C. Sleep and rheumatologic disorders. Sleep Med Rev 2008; 12:211-28.

3. Wilcox S, Brenes GA, Levine D, Sevick MA, Shumaker SA, Craven T. Factors related to sleep disturbance in older adults experiencing knee pain or knee pain with radiographic evidence of knee osteoarthritis. J Am Geriatr Soc 2000;48:1241-51.
4. Sasaki E, Tsuda E, Yamamoto Y, Maeda S, Inoue $\mathrm{R}$, Chiba $\mathrm{D}$, et al. Nocturnal knee pain increases with the severity of knee osteoarthritis, disturbing patient sleep quality. Arthritis Care Res (Hoboken) 2014;66:1027-32.

5. Verhagen A, Bierma-Zeinstra S, Lambeck J, Cardoso $\mathrm{JR}$, de Bie R, Boers $\mathrm{M}$, et al. Balneotherapy for osteoarthritis. A cochrane review. J Rheumatol 2008;35:1118-23.

6. Kellgren JH, Lawrence JS. Radiological assessment of osteo-arthrosis. Ann Rheum Dis 1957;16:494-502.

7. Bellamy N, Buchanan WW, Goldsmith $\mathrm{CH}$, Campbell J, Stitt LW. Validation study of WOMAC: a health status instrument for measuring clinically important patient relevant outcomes to antirheumatic drug therapy in patients with osteoarthritis of the hip or knee. J Rheumatol 1988;15:1833-40.

8. Buysse DJ, Reynolds CF 3rd, Monk TH, Berman SR, Kupfer DJ. The Pittsburgh Sleep Quality Index: a new instrument for psychiatric practice and research. Psychiatry Res 1989;28:193-213.

9. Roemer FW, Kassim Javaid M, Guermazi A, Thomas M, Kiran A, Keen R, et al. Anatomical distribution of synovitis in knee osteoarthritis and its association with joint effusion assessed on non-enhanced and contrast-enhanced MRI. Osteoarthritis Cartilage 2010;18:1269-74.

10. Wigler I, Elkayam O, Paran D, Yaron M. Spa therapy for gonarthrosis: a prospective study. Rheumatol Int 1995;15:65-8.

11. Odabasi E, Turan M, Erdem H, Tekbas F. Does mud pack treatment have any chemical effect? A randomized controlled clinical study. J Altern Complement Med 2008;14:559-65.

12. Matz H, Orion E, Wolf R. Balneotherapy in dermatology. Dermatol Ther 2003;16:132-40.

13. Bender T, Karagülle Z, Bálint GP, Gutenbrunner C, Bálint PV, Sukenik S. Hydrotherapy, balneotherapy, and spa treatment in pain management. Rheumatol Int 2005;25:220-4.

14. Jezová D, Vigas M, Tatár P, Jurcovicová J, Palát $\mathrm{M}$. Rise in plasma beta-endorphin and $\mathrm{ACTH}$ in response to hyperthermia in sauna. Horm Metab Res 1985;17:693-4.

15. Cozzi F, Lazzarin P, Todesco S, Cima L. Hypothalamicpituitary-adrenal axis dysregulation in healthy subjects undergoing mud-bath applications. Arthritis Rheum 1995;38:724-6.

16. Foley D, Ancoli-Israel S, Britz P, Walsh J. Sleep disturbances and chronic disease in older adults: results of the 2003 National Sleep Foundation Sleep in America Survey. J Psychosom Res 2004;56:497-502.

17. Smith MT, Haythornthwaite JA. How do sleep disturbance and chronic pain inter-relate? Insights from the longitudinal and cognitive-behavioral clinical trials literature. Sleep Med Rev 2004;8:119-32.

18. Haack M, Sanchez E, Mullington JM. Elevated inflammatory markers in response to prolonged 
sleep restriction are associated with increased pain experience in healthy volunteers. Sleep 2007;30:1145-52.

19. Smith MT, Edwards RR, McCann UD, Haythornthwaite JA. The effects of sleep deprivation on pain inhibition and spontaneous pain in women. Sleep 2007;30:494-505.

20. Vitiello MV, Rybarczyk B, Von Korff M, Stepanski EJ. Cognitive behavioral therapy for insomnia improves sleep and decreases pain in older adults with co-morbid insomnia and osteoarthritis. J Clin Sleep Med 2009;5:355-62.

21. Rybarczyk B, DeMarco G, DeLaCruz M, Lapidos $\mathrm{S}$, Fortner B. A classroom mind/body wellness intervention for older adults with chronic illness: comparing immediate and 1-year benefits. Behav Med 2001;27:15-27.

22. Astin JA, Beckner W, Soeken K, Hochberg MC, Berman B. Psychological interventions for rheumatoid arthritis: a meta-analysis of randomized controlled trials. Arthritis Rheum 2002;47:291-302.

23. KE, Keefe FJ, Scipio CD, Perri LM, Abernethy AP. Psychological interventions for arthritis pain management in adults: a meta-analysis. Health Psychol 2007;26:241-50.

24. Roehrs T, Hyde M, Blaisdell B, Greenwald M, Roth T. Sleep loss and REM sleep loss are hyperalgesic. Sleep 2006;29:145-51. 